\title{
Hernia inguinal bilateral en 100 pacientes manejados con técnica laparoscópica transabdominal preperitoneal (TAPP)
}

\author{
Transabdominal preperitoneal technique (TAPP) for the \\ treatment of 100 patients with bilateral groin hernia \\ Ernesto Manuel Góngora Gómez* \\ * Hospital General del Centro Médico Nacional «La Raza». Instituto Mexicano del Seguro Social.
}

\section{RESUMEN}

Introducción: La hernia inguinal bilateral (HIB) en adultos tiene una prevalencia de $17-35 \%$ que se incrementa con la edad. Después de los 50 años, la prevalencia de hernia inguinal sincrónica puede ser de $35 \%$ o desarrollarse en forma metacrónica hasta en $60 \%$, según avance la edad. Las técnicas TAPP (transabdominal preperitoneal) y TEP (totalmente extraperitoneal) son electivas para el manejo de la HIB, pero TAPP facilita la realización de cirugías intraabdominales concomitantes y permite identificar la hernia contralateral sincrónica no estudiada. Después de TAPP o TEP es frecuente la sensación de «un bulto» inguinal. Se propuso la resección de todos los lipomas herniarios y se dio seguimiento puntual por 18 meses para dilucidar si fuera ésta una de las causalidades. Material y métodos: Estudio de serie de casos, observacional, prospectivo, descriptivo. De enero 2009 a marzo 2020 se sometió a TAPP a 100 adultos con HIB, intervenidos por el mismo cirujano y equipo. Se describe sucintamente la técnica, se informan diversas variables. Resultados: Edad promedio, 48.1 años; peso, $73.9 \mathrm{~kg}$; comorbilidades, 59 pacientes; tiempo operatorio, 118 minutos; rango de sangrado, 5-150 mL; posoperatorio, 1.6 días; complicaciones menores, 35; sensación de «bulto» posoperatorio, 19. TAPP es la técnica de elección para el manejo de la HIB por ofrecer múltiples ventajas ratificadas en la bibliografía. La hernia sincrónica contralateral puede hallarse hasta en el $52 \%$ de los casos. Conclusiones: Es pertinente indagar intencionadamente la hernia contralateral sincrónica. TAPP es primera elección para la HIB, incluyendo las circunstancias que ameriten cirugía intraabdominal concomitante. Deben extirparse los lipomas herniarios al operar hernias inguinales.

Palabras clave: Hernia inguinal bilateral, TAPP, TEP, bulto inguinal, cirugía intraabdominal concomitante.

\section{ABSTRACT}

Introduction: Bilateral groin hernia (BGH) in adults has a prevalence of $17-35 \%$ that increases with age. In people older than 50 years, synchronic groin hernias prevalence can be as high as $35 \%$ or become a metachronic hernia up to $60 \%$ as age advances. TAPP (transabdominal preperitoneal) and TEP(totally extraperitoneal)are elective techniques for $B G H$ repair, however, TAPP makes it easier to carry out other concomitant intra-abdominal surgeries, and allows the identification of unnoticed synchronic contralateral hernias. After TAPP or TEP, patients often have the sensation of a groin lump or swelling in the groin area. Therefore, in the present research, resection of all hernial lipomas was proposed, as well as a punctual follow-up during the subsequent 18 months in order to elucidate whether this was one of the causes. Material and methods: Observational, prospective and descriptive case series study, from January 2009 to March 2020; 100 adults with BGH underwent TAPP, operated on by the same surgeon and his team. The technique is succinctly described and different variables are reported. Results: Average age, 48.1 years; weight, $73.9 \mathrm{~kg}$; comorbidities, 59 patients; operating time, 118 minutes; bleeding 5-150 mL; hospital stay, 1.6 days; minor complications, 35; sensation of groin lump, 19. TAPP has become the technique of choice in repairing $B G H$ due to its multiple advantages ratified in the literature. The synchronic contralateral groin hernia can be found in up to $52 \%$ of cases. Conclusions: It is relevant to inquire about synchronic contralateral groin hernia. TAPP is the gold standard in BGH repair, including the cases needing concomitant intra-abdominal surgeries. All hernial lipomas must be resected when operating on groin hernias.

Keywords: Bilateral groin hernia, TAPP, TEP, groin lump, concomitant intra-abdominal surgery.

\section{Recibido: 07/07/2020. Aceptado: 05/08/2020. \\ Correspondencia: Dr. Ernesto Manuel Góngora Gómez \\ E-mail: herniagongora@gmail.com}

Citar como: Góngora GEM. Hernia inguinal bilateral en 100 pacientes manejados con técnica laparoscópica transabdominal preperitoneal (TAPP). Rev Mex Cir Endoscop. 2020; 21 (3): 126-132. https://dx.doi.org/10.35366/99835 


\section{INTRODUCCIÓN}

La posibilidad de que un adulto sufra de una hernia inguinal, a lo largo de su vida, tiene una incidencia de $17 \%$; sin embargo, ésta se incrementa hasta 35\% después de los 50 años. ${ }^{1,2}$ Es a partir de esta etapa de la adultez cuando la prevalencia de la hernia inguinal bilateral (HIB) inicia su incremento, aunque cabe afirmar que no se tiene bien definido el índice de esta prevalencia en el mundo.

La aparición de una hernia inguinal modifica, en menor o mayor grado, la calidad de vida, afectando las actividades laboral, sexual y deportiva. Altera la autoestima, suele provocar dolor transitorio y/o recurrente hasta en $80 \%$ de los pacientes que la sufren. Puede desencadenar encarcelamiento en $7 \%$ de los casos o estrangulamiento en hasta $3 \%$. Cuando un adulto mayor, que sufre dos o más comorbilidades, padece un episodio de estrangulamiento herniario, la mortalidad puede llegar a ser de hasta $40-50 \%{ }^{3}$

En este cada vez mayor grupo etario, la aparición de una hernia contralateral sincrónica oculta está en el índice de 35\%. Asimismo, se ha descubierto que la posibilidad de que esa hernia se manifieste como una hernia metácrona en los próximos siete años o más será 60 veces mayor. ${ }^{4-7}$

$\mathrm{Si}$ al sufrir hernia inguinal unilateral se tienen estos peligros, ¿será posible que tales riesgos se dupliquen al aparecer una hernia inguinal metácrona en la ingle contralateral?

Estas son razones para investigar la probable sincronía herniaria al momento del diagnóstico y proponer la reparación quirúrgica bilateral en dichas edades, puntualizando que la incidencia de la hernia metacrónica se incrementa conforme avanza la existencia.

Desde el inicio del presente siglo se ha sugerido que se deben corregir quirúrgicamente las hernias inguinales al momento del diagnóstico en todo paciente adulto $y$, mayormente indicado, si tiene 50 años o más..$^{3-7}$

Está demostrado que la reparación laparoscópica transabdominal preperitoneal (TAPP por sus siglas en inglés: transabdominal preperitoneal) se ha convertido en el procedimiento de elección para resolver los casos de HIB, ${ }^{1,2,8}$ complementando además, que TAPP (transabdominal preperitoneal) permite resolver otras patologías concomitantes intraabdominales, como son: colecistectomías, hernias hiatales y de pared, trastornos ginecológicos, urológicos, etcétera. ${ }^{9}$ Se propone de igual manera esta técnica, como adecuada elección para el manejo de la HIB en los casos que presentan recurrencia o multirrecurrencia, particularmente si la primer cirugía se realizó por vía anterior. 2,4,10-13 Por medio de TAPP es posible identificar, durante el transoperatorio, una hernia contralateral sincrónica oculta no investigada o no evidenciada por la clínica o por imagen. .,7 $^{-7}$
Ha sido ampliamente demostrado que las plastias inguinales «bajo tensión» han caído en desuso, a excepción de la técnica Shouldice, y que el índice de recurrencia, al operar «sin tensión» con las técnicas de Lichtenstein o Mesh-Plug, es prácticamente el mismo $(0.5-2.5 \%)^{14}$ que operando con las técnicas laparoscópicas TAPP o TEP (Totally Extraperitoneal); ${ }^{10}$ las técnicas laparoscópicas ofrecen otras ventajas sobre aquéllas: recuperación física y laboral más rápida, menor dolor y consumo de analgésicos en el posoperatorio, mejores resultados cosméticos y mayor satisfacción personal.

Se ignora cuántos pacientes, después de ser sometidos a TAPP o TEP, se quejan de la sensación de un «bulto», que frecuentemente se asocia a dolor, en la ingle operada. Cuando existe la queja, se debe estudiar mediante una exploración física detallada complementada por un ultrasonido $y$, si se asocia a dolor puede exigir, incluso, el realizar un escrutinio de mapeo por dermatomas.

Se ha planteado que la sensación o evidencia de «bulto» inguinal posoperatorio puede ser a consecuencia de:

1. La formación de un seroma, que por lo general es secundario a la presencia de la malla (cuerpo extraño), mismo que se auto reabsorbe en pocos días y, consecutivamente, esa sensación de «bulto» desaparece.

2. No resecar los lipomas herniarios. Respecto a esto, no hay evidencia bibliográfica que sustente esta afirmación. Sin embargo, no son pocos los pacientes que emiten esta queja después de una inguinoplastia por laparoscopía; incluso en muchos de ellos es posible palpar el «bulto lipomatoso» en la región inguinal después de la cirugía.

Con base en esta consideración, se definió como uno de los objetivos del presente trabajo el identificar y extirpar los lipomas herniarios durante el acto quirúrgico en todos los pacientes atendidos, a fin de darles puntual seguimiento después de la cirugía.

La ejecución de este estudio tuvo tres objetivos:

1. Informar los resultados de someter a la técnica laparoscópica TAPP a 100 pacientes en los que se documentó hernia inguinal bilateral.

2. Dilucidar si el extirpar el lipoma herniario influye o no en la aparición posoperatoria de la sensación de «bulto inguinal posoperatorio».

3. Ratificar que TAPP permite y facilita en el mismo paciente la resolución de otras patologías concomitantes por medio de cirugías laparoendoscópicas, al tiempo de resolver la HIB. 


\section{MATERIAL Y MÉTODOS}

Diseño: estudio de serie de casos, observacional, prospectivo, descriptivo.

De enero de 2009 a marzo de 2020, se documentó el diagnóstico de HIB en 100 pacientes adultos, mediante estudio clínico, complementado con ultrasonido de ambas regiones inguinales o con tomografía axial computarizada de abdomen.

Se le propuso a cada paciente someterlo a cirugía laparoscópica tipo TAPP con el fin de corregir ambas hernias inguinales, aplicando una malla de polipropileno en las regiones preperitoneales de ambas regiones inguinales. Se elaboró una hoja de consentimiento informado a ser firmado por cada individuo.

El escrutinio diagnóstico se realizó en 123 pacientes, sin embargo, 23 de éstos se excluyeron: cinco pacientes por cursar con, al menos, una hernia inguinoescrotal mayor a $15 \mathrm{~cm}$ de longitud, realizando la métrica en decúbito. Diez pacientes aceptaron someterse a plastia TAPP, solamente unilateral. En dos ocasiones se sugirió postergar la cirugía hasta corregir trastornos cardiológicos que la contraindicaban. Los otros seis no aceptaron ningún procedimiento con nuestro equipo. Se excluyeron los pacientes que tenían IMC de 35 o mayor.

Todos los pacientes se sometieron a estudios laboratoriales de rutina preoperatorios. A los mayores de 40 años se les practicó, además, radiografía del tórax, electrocardiograma y evaluación por medicina interna.

Todos los pacientes fueron intervenidos por el mismo cirujano y con la misma técnica operatoria en hospitales privados de la Ciudad de México, realizando plastia inguinal por laparoscopía transabdominal preperitoneal (TAPP) convencional, bajo anestesia general, realizando el neumoperitoneo con aguja de Veress, instalando tres puertos de trabajo, introduciendo trocares con navaja, uno de $12 \mathrm{~mm}$ a nivel umbilical y dos de $5 \mathrm{~mm}$ en ambos flancos a la misma altura del ombligo. Se utilizó un lente de visión de $5 \mathrm{~mm}$ con angulación de 30 grados. En todos los casos se usó energía monopolar para corte y disección. Se realizó apertura de la ventana peritoneal retroinguinal logrando exponer el área de visión crítica del orificio miopectíneo de Fruchaud, hasta visualizar: por arriba, el músculo transverso; medialmente, por dentro de la línea que demarca el ligamento de la arteria umbilical ipsilateral; lateralmente, a dos centímetros abajo de la espina iliaca anterosuperior; por abajo, hasta $4-5 \mathrm{~cm}$ de la cintilla ileopectínea. Se visualizó el ligamento de Cooper y los orificios femoral y obturatriz. Se retrajo el saco herniario. Se hizo una detallada búsqueda para identificar y extirpar el lipoma herniario que, en la gran mayoría de los casos, se desliza a través del orificio herniario y discurre hacia afuera del piso inguinal. En las situaciones de hernias directas, se retrae el saco de fascia transversalis y se lo fija con una o dos grapas al Cooper y así se logra colapsar ese saco y minimizar la formación de seroma. ${ }^{15}$ Se aplicó malla de polipropileno con dimensiones de $10 \times 13$ y/o hasta $14 \times 16 \mathrm{~cm}$, fijándola con grapas metálicas o absorbibles, una grapa al Cooper y de dos a tres al transverso. Se cerró el peritoneo con sutura de lenta absorción. En algunas ocasiones se instaló drenaje cerrado en la región preperitoneal extrayéndolo por el puerto ipsilateral. Se afrontó el borde aponeurótico del puerto del ombligo con sutura de lenta absorción. Se realizaron diversos procedimientos laparoendoscópicos concomitantes durante el mismo acto quirúrgico. Los pacientes fueron egresados uno o más días después, según su evolución. Se analizaron diversas variables que se describen en los resultados. Se dio seguimiento posoperatorio por 18 meses.

\section{RESULTADOS}

Se trató a 100 pacientes con hernia inguinal bilateral con diagnóstico confirmado por imagen.

Se analizaron las variables de edad, sexo, peso y comorbilidades, como se muestra en la Tabla 1.

Desde el escrutinio inicial, se pudo descubrir que 52 de los 100 pacientes no sabían que tenían una hernia sincrónica contralateral, en 31 de ellos sí se sospechó durante la exploración inicial y en los restantes 21 no se tuvo esta

\begin{tabular}{lcc} 
& Tabla 1: Variables. & \\
& & Rango \\
\hline Edad (años) & 48.1 & $17-72$ \\
Sexo & & \\
Hombres ( $\mathrm{n})$ & 84.0 & \\
Mujeres ( $)$ & 16.0 & $51-104$ \\
Peso (kg) & 73.9 & \\
Comorbilidades & & \\
Tabaquismo-EPOC & 35.0 & \\
Sobrepeso & 25.0 & \\
Diabetes mellitus & 6.0 & \\
HTA & 10.0 \\
Prostatismo & 11.0 \\
ERGE & 7.0 \\
Colecistopatía & 10.0 \\
Quiste ovárico & 1.0 \\
Hernia umbilical & 19.0 \\
Hernia ventral & 2.0 \\
\hline EPOC = enfermedad pulmonar obstructiva crónica, HTA = hipertensión \\
arterial, ERGE = enfermedad por reflujo gastroesofágico.
\end{tabular}




\section{Tabla 2: Variables.}

\begin{tabular}{|c|c|c|}
\hline \multicolumn{3}{|l|}{ Malla de polipropileno } \\
\hline $\begin{array}{l}\text { Peso medio, confeccionada } \\
\text { en quirófano }\end{array}$ & 36 & \\
\hline $\begin{array}{l}\text { Peso medio, manufactura } \\
\text { industrial }\end{array}$ & 46 & \\
\hline $\begin{array}{l}\text { Peso ligero, manufactura } \\
\text { industrial }\end{array}$ & 18 & \\
\hline \multicolumn{3}{|l|}{ Sangrado operatorio TAPP } \\
\hline$<5 \mathrm{~cm}^{3}$ & 51 casos & \\
\hline$>5 \mathrm{~cm}^{3}$ & 49 casos & $27 \mathrm{~cm}^{3}$ \\
\hline \multicolumn{3}{|l|}{ Drenaje cerrado } \\
\hline Tipo Drenovack & 9 & \\
\hline Tipo Blake & 2 & \\
\hline Tiempo operatorio TAPP & $\begin{array}{c}118 \\
\text { minutos }\end{array}$ & $\begin{array}{l}85-240 \\
\text { minutos }\end{array}$ \\
\hline Conversión & $\begin{array}{c}1 \text { caso a } \\
\text { Lichtenstein }\end{array}$ & \\
\hline Estancia hospitalaria & 1.6 días & \\
\hline Seguimiento & 18 meses & \\
\hline \multicolumn{3}{|l|}{ Complicaciones } \\
\hline Inguinodinia & 17 & \\
\hline Hematoma & 9 & \\
\hline Hidrocele & 4 & \\
\hline Varicocele & 2 & \\
\hline Orquitis & 2 & \\
\hline Infección & 1 & \\
\hline Queja de "bulto" inguinal & 19 & \\
\hline
\end{tabular}

TAPP $=$ transabdominal preperitoneal.

sospecha; sin embargo, en el total de los 52, se confirmó la existencia de dicha sincronía herniaria al realizar ultrasonido o tomografía.

Es importante destacar que 27 hernias confirmadas mediante ultrasonido no presentaron identificación visual de saco peritoneal al momento de realizar la exploración laparoscópica transoperatoria, pero sí se confirmó, al exponer el orificio miopectíneo de Fruchaud, que el componente herniario era un lipoma de menor o mayor tamaño.

Los casos diagnosticados por ultrasonido fueron 98 y dos mediante TAC, mientras que la evolución promedio de la hernia predominante fue de 1.7 años.

En la Tabla 2 se analizan las variables: tipo de maIla aplicada, sangrado transoperatorio, tipo de drenaje instalado, tiempo operatorio durante TAPP, número de conversiones, tiempo de estancia hospitalaria, seguimiento y complicaciones.

En la Tabla 3 se exponen el tipo de hernia, por su aparición, en primarias y recurrentes; por su comportamiento clínico, en encarceladas y estranguladas; por su composición inguinoescrotal, según la clasificación de Stromeyer $y$, finalmente, por sus dimensiones.
El número de cirugías concomitantes se describe en la Tabla 4.

Es altamente destacable que en la presente muestra, de 100 pacientes, sólo el 48\% de los casos se presentaron a consulta médica quejándose de sufrir HIB, pero que después de un escrutinio clínico y por imagen, se descubrió que el 52\% restante tenían una hernia contralateral sincrónica.

La incidencia de la hernia inguinal sincrónica reportada en el presente estudio es más alta que la de otros trabajos, ${ }^{4,6,8}$ por lo que se considera pertinente proponer el estudio sistemático de la bilateralidad herniaria inguinal en todo adulto diagnosticado con hernia inguinal unilateral que tenga 50 años o más.

No se ha definido cuántos pacientes mayores de 50 años, a los que se les ha realizado inguinoplastia unilateral, podrán desarrollar en el futuro una hernia contralateral, pero será interesante evaluar la repercusión de tener que operarse varios años después o, eventualmente, enfrentarse a un episodio de estrangulamiento herniario, aduciendo que éste se presenta, principalmente, después de los 60 años y que su desenlace es mayormente grave, si el paciente padece más de una comorbilidad.

La edad promedio de los pacientes, en este estudio, fue 48.1 años con rango de 17 a 74 . Un paciente tenía 17 años y otros nueve tenían 30 años o menos. Los 90 pacientes restantes tenían un promedio de 50.1 años con rango de 31 a 74 años (Tabla 1).

La comorbilidad preoperatoria en tales casos es alta, ya que 59 de los cien pacientes presentaron una o más patologías, destacando la asociación de sobrepeso, diabetes, hipertensión y tabaquismo-EPOC en 22 de ellos. Se puntualiza que no se incluyó, en la casuística utilizada, a ningún paciente con gran obesidad (IMC mayor a 35) y que se les obligó a suspender el tabaquismo, al menos 30 días antes de la cirugía y a compensar sus niveles de tensión arterial y glucemia, al menos 15 días antes de la cirugía (Tabla 1).

El sangrado operatorio en el procedimiento TAPP fue menor a $5 \mathrm{~mL}$ en 51 pacientes y en los 49 restantes, el sangrado promedió $27 \mathrm{~mL}$ Tabla 2. La decisión de dejar drenaje cerrado obedeció a:

1. Volumen de sangrado

2. Tiempo operatorio

3. Complejidad técnica del caso:
a. Hernias encarceladas
b. Hernias inguinoescrotales
c. Hernias estranguladas
d. Hernias recurrentes
e. La existencia de grandes lipomas herniarios 
El tiempo de estancia hospitalaria fue de 1.6 días; no difiere del reportado en la literatura. . $^{6,7,9,16}$ En los casos en los que se realizó cirugía concomitante, el tiempo promedio se elevó a 1.9 días.

Se dio seguimiento durante 18 meses con visitas presenciales a la semana, al primer y al segundo mes. Después, al sexto, doceavo y dieciochoavo mes mediante entrevista telefónica (Tabla 2). No se identificó recurrencia herniaria durante los 18 meses de seguimiento.

La queja de inguinodinia posoperatoria fue temporal en los 17 pacientes que la sufrieron. Se manejaron conservadoramente según la causa y ninguno de ellos manifestó su permanencia después del tercer mes.

De los cuatro eventos de hidrocele, tres de ellos se resolvieron mediante una o dos punciones evacuadoras. Uno de ellos no se resolvió, persiste después de tres años. El paciente se rehúsa a su manejo quirúrgico.

Un paciente, que había sido sometido a plastia Lichtenstein dos años antes y sufrió recurrencia, presentó varicocele después de la intervención con plastia TAPP. El segundo caso que presentó varicocele, lo manifestó a partir del tercer mes de la plastia TAPP. No fue posible dilucidar si ocurrió a consecuencia de la cirugía.

Ocurrieron dos eventos de orquitis, ambos dentro del primer mes después la cirugía y se resolvieron con manejo conservador.

Se tuvo una circunstancia de infección de sitio quirúrgico que obligó al retiro de la malla mediante abordaje por vía anterior. En este suceso se reforzó el piso inguinal con técnica de Shouldice. Se le dio seguimiento durante 30 meses sin identificar recurrencia.

De las 200 hernias operadas, 185 eran primarias y 15 recurrentes, todas estas operadas previamente por vía anterior sin saberse, al detalle, cuántas de éstas fueron con o sin tensión. Se presentaron encarceladas 18 hernias (irreductibles sin trastornos circulatorios). El promedio de edad de estos casos fue de 50.4 años. Se tuvo sólo un evento de hernia estrangulada (irreductible con alteraciones circulatorias) ratificada por TAC, que confirmó la sincronía herniaria contralateral; este paciente tenía 62 años. No requirió resección intestinal (Tabla 3).

Cursaron con hernia inguinoescrotal 16 pacientes. En 11 de ellos la hernia se encontraba encarcelada y en cinco la hernia era reductible. El promedio de edad de estos pacientes fue de 75.8 años. Según la clasificación de Stromeyer, fueron las hernias directas las más numerosas; las menos fueron femorales, todas estas ocurrieron en mujeres. Se identificaron, en todos los pacientes, la hernia de mayor dimensión o predominante y la de menor tamaño, 76 casos tenían hernias menores a $8 \mathrm{~cm}$ y otros 24 mayores a $8 \mathrm{~cm}$ (Tabla 3).

\section{DISCUSIÓN}

Las complicaciones de recurrencia e inguinodinia se constituyeron como los principales parámetros para medir los niveles de éxito de la inguinoplastia unilateral hasta finales del siglo pasado, en función de que ambas complicaciones tenían una incidencia moderadamente alta; sin embargo, con la llegada de las nuevas técnicas laparoscópicas se modificó la métrica y se agregaron otros indicadores que miden también esos niveles de éxito: infección de sitio quirúrgico, hidrocele, sensación remanente de bulto inguinal posoperatorio, insatisfacción personal, resultados cosméticos. ${ }^{16,17}$

Queda claro que cualquier técnica operatoria para la HIB debiera presentar baja incidencia en todos estos indicadores.

En la época de la cirugía «con tensión» existía contraindicación relativa para operar ambas hernias el mismo día debido a la alta incidencia de dolor, recurrencia y lenta recuperación que se tenían como resultado. Con la llegada

\section{Tabla 3: Clasificación de hernias.}

$\begin{array}{lclr}\text { Hernias primarias } & 185 & & \\ \text { Hernias recurrentes } & 15 & \text { Con una cirugía previa } & 12 \\ & & \text { Con dos cirugías previas } & 2 \\ \text { Hernias encarceladas } & & \text { Con tres cirugías previas } & 1 \\ & 18 & \text { Saco herniario }<5 \mathrm{~cm} & 3 \\ \text { Hernias estranguladas } & 1 & \text { Saco herniario }>5 \mathrm{~cm} & 15 \\ \text { Hernia inguinoescrotal } & 16 & \text { Reductibles } & 5 \\ & & \text { Encarceladas } & 11 \\ \text { Clasificación Stromeyer } & & \text { Indirectas } & 14 \\ & & \text { Directas } & 53 \\ & & \text { Mixtas } & 28 \\ \text { Dimensión de las hernias } & \text { Femorales } & 5 \\ & & <\text { de } 8 \mathrm{~cm} & 176 \\ & & >\text { de } 8 \mathrm{~cm} & 24\end{array}$


Tabla 4: Cirugías concomitantes. $\mathbf{N}=\mathbf{4 0}$.

\begin{tabular}{lc} 
& $\mathrm{n}$ \\
\hline Funduplicaturas & 7 \\
Colecistectomías & 10 \\
Umbiliplastias & 19 \\
Plastia ventral & 2 \\
Resección de quiste de ovario & 1 \\
Oclusión tubaria bilateral & 1 \\
\hline
\end{tabular}

de las técnicas abiertas sin tensión, se abatió el índice de recurrencia, mejoró el de lenta recuperación, pero no el de dolor, manteniéndose en cierta controversia la inguinoplastia bilateral en un solo tiempo.

Se han publicado algunos trabajos que le dan mayor confiabilidad a la técnica laparoscópica TAPP para reparar la HIB de manera sincrónica que a las técnicas antes referidas, e incluso, que a la técnica totalmente extraperitoneal (TEP). ${ }^{2}$ Esta preferencia puede sustentarse en el análisis de lo siguiente:

1. Atenúa el dolor posoperatorio en tiempo e intensidad.

2. Disminuye el consumo de analgésicos en el posoperatorio.

3. Reincorpora tempranamente al paciente a sus labores o a sus actividades rutinarias.

4. Aminora el índice de recurrencias.

5. Decrece el índice de inguinodinia.

6. Reduce la estancia hospitalaria.

7. Consigue mejores resultados cosméticos.

8. Alcanza mejores índices de satisfacción personal posoperatoria.

9. Facilita la realización de otras cirugías laparoendoscópicas concomitantes.

Continúa dilucidándose sobre la epidemiología de la HIB y las propuestas actuales para su manejo. Cada vez más estudios hablan sobre la incidencia de esta patología e investigan sobre sus posibles causalidades: herniosis, tabaquismo, enfermedades de la colágena, enfermedad aórtico-aneurismática abdominal, influencia genética, senescencia, diabetes, obesidad, síndrome metabólico, etcétera. También se encuentra en estudio el definir qué tan frecuentemente aparece la hernia contralateral sincrónica al momento de diagnosticar la primera, o bien, pronosticar cuánto tiempo después aparecerá la hernia contralateral metácrona.

No se tiene información documentada en la literatura sobre la ocasional percepción posoperatoria de tumoración o «bulto» o «hernia lipomatosa» en la región inguinal, no obstante, aquí se decidió investigar esta aparente complicación de las técnicas laparoscópicas con base en la propuesta de extirpar todos los lipomas herniarios e indagar sobre esta queja después de la cirugía.

A lo largo del seguimiento posoperatorio, se interrogó a todos los pacientes sobre la sensación de «bulto en las ingles»; 19 de ellos se quejaron temporalmente de tal sensación. Se les practicó un ultrasonograma. En 15 de ellos se identificó la presencia de colección líquida. Uno más fue el que hubo de convertirse a técnica Lichtenstein, identificando conglomerado ganglionar inflamatorio. Dos pacientes fueron de los que presentaron hidrocele, que acabó resuelto mediante punción evacuadora. En el último de los 19, no se logró identificar la causa mediante el ultrasonido. En todos los pacientes la sensación de «bulto» desapareció en corto plazo y ninguno de ellos la refirió después del tercer mes, ni a lo largo de los 18 meses que se les dio seguimiento.

Resecar los lipomas herniarios ocupó de dos a 15 minutos, según las circunstancias, motivo que alargó el tiempo operatorio, pero a la vez permitió obtener los resultados descritos y así poder darle valor a la decisión de extirpar, siempre, los lipomas herniarios.

Vale resaltar que 27 casos en los que no se identificó saco herniario peritoneal, sí tenían una «hernia lipomatosa». Ese lipoma es el que discurría, desde la región preperitoneal, a través del orificio herniario para posicionarse en la región anterior o externa del piso inguinal.

La técnica TAPP, en esta serie de casos, permitió realizar, en el mismo acto quirúrgico, 40 cirugías miniinvasivas diversas, como se describe en la Tabla 4, confirmando así su gran compatibilidad con la multicirugía intraabdominal concomitante.

\section{CONCLUSIONES}

La incidencia de la hernia inguinal bilateral es baja, pero va creciendo conforme la población nacional va teniendo una expectativa de vida más prolongada. Tal incidencia crecerá más aún si se aplica un escrutinio escrupuloso en los adultos mayores a los 50 años.

A lo largo de 11 años se integró la presente casuística, permitiendo ratificar, frente a la bibliografía internacional, el resultado de algunas variables, pero también complementar la información de algunas otras que conceden enunciar estas conclusiones:

1. Indagar intencionadamente, auxiliándose con estudios de imagen, en la región inguinal contralateral, en todo paciente adulto al que se le diagnostique hernia inguinal unilateral. 
2. Proponer reparación herniaria bilateral mediante la técnica TAPP en todo paciente de 50 años o mayor, al momento de confirmar el diagnóstico de HIB.

3. Proceder mediante TAPP para reparar la HIB en caso de que el paciente requiera, además, otro tipo de cirugía de mínimo acceso intrabdominal concomitante.

4. Plantear la técnica TAPP como el procedimiento de elección en la reparación de la HIB.

5. Identificar y extirpar todos los lipomas herniarios al operar las hernias inguinales.

\section{REFERENCIAS}

1. Guías de práctica clínica para hernias de la pared abdominal. AMH. 2015; 1-49.

2. The Hernia Surge Group: International guidelines for groin hernia management. Hernia. 2018; 22: 1-165.

3. Góngora EM. La hernia inguinal estrangulada. Cir Cir. 2012; 80: 357-367.

4. Bochkarev V, Rimgley C, Vitamvas M, Olegnikov D. Bilateral laparoscopic inguinal hernia repair in patients with occult contralateral inguinal defects. Surg Endosc. 2007; 21: 734-736.

5. Alarcón A, Tinoco I, Socas M. Tallón L, Morales S. Hernia inguinal bilateral por laparoscopía. Cir Andal. 2018; 29: 110-111.

6. Saggar VR, Sarangi R. Occult hernias and bilateral endoscopic total extraperitoneal inguinal hernia repair: Is there a need for prophylactic repair? Hernia. 2007; 11: 47-49.

7. García-Moreno JL, Ruiz-Lupiáñez E, Suárez-Grau JM. Tipos de reparaciones actuales (anatómicas y protésicas). Cir Andal. 2018; 29: 91-93.
8. Simons MP, Anfenaker T, Bag-Nielsen M, Bouillot JL, Campanelli G, Conze J et al. European Hernia Society Guidelines on the treatment of inguinal hernia in adult patients. Hernia. 2009; 13: 343-403.

9. Acuña J, Elles C, Espitia B, Martínez E. Carrasquilla R. Alcalá R, Manjarréz A. Hernioplastias inguinales por vía laparoscópica. Experiencia inicial en 324 casos. Rev Hispanoam Hernia. 2016; 4: 87-93

10. Gould J. Laparoscopic versus open inguinal hernia repair. Surg Clin North Am. 2008; 88: 1073-1081.

11. Weber A, Weber P, Garteiz D. Laparoscopy and bilateral inguinal hernias. J Surg Transplant Sci. 2016; 4: 1019.

12. Mohamed-El-Barbary H, Ahmed-Nazeer N. Laparoscopic TAPP repair for bilateral inguinal hernia, single large mesh versus double mesh technique. J Am Science. 2017; 13: 55-61.

13. Weber SA, Arguellez LP, Garteiz MD, Vega RF, Carbo RR. Experiencia de 10 años con malla de polipropileno en la reparación de la hernia inguinal en el Hospital Ángeles Lomas. Rev Mex Cir Endoscop. 2011; 12: 118122.

14. Takayama Y, Kaneoka Y, Maeda A, Takahashi T. Uji M. Laparoscopic transabdominal preperitoneal repair versus open mesh plug repair for bilateral inguinal hernia. Ann Gastroenterol Surg. 2020; 4: 156-162.

15. Gómez M. Cirugía laparoscópica de la hernia inguinal: TEP. Cir Andal. 2018; 29: 174-177.

16. Aragón F, Expósito M, Hernández L, Aragón L. Hernioplastia laparoscópica y reparación anterior sin tensión: ¿Qué dicen las evidencias? Rev Cubana Cir. 2009; 48.

17. Lokhart K, Dunn D, Teo S, Yng I, Dhillon M, Teo E et al. Mesh versus non mesh for inguinal and femoral hernia repair. Cochrane Database Syst Review. 2018; 9: CD011517. 